\title{
Freeze-like responses to pain in humans and its modulation by social context
}

\author{
Kai Karos ${ }^{\text {Corresp., } 1,2}$, Ann Meulders ${ }^{2,3}$, Tine Leyssen ${ }^{3}$, Johan W Vlaeyen ${ }^{2,3}$ \\ ${ }^{1}$ Centre for the Psychology of Learning and Experimental Psychopathology, Faculty of Psychology and Educational Sciences, KU Leuven, Leuven, \\ Vlanders, Belgium \\ 2 Research Group on Experimental Health Psychology, Department for Clinical Psychological Science, Faculty of Psychology and Neuroscience, Maastricht \\ University, Maastricht, Limburg, Netherlands \\ Research Group on Health Psychology, Faculty of Psychology and Educational Sciences, KU Leuven, Leuven, Vlanders, Belgium \\ Corresponding Author: Kai Karos \\ Email address: kai.karos@kuleuven.be
}

Background. Maladaptive defensive responses such as excessive avoidance behavior have received increasing attention as a main mechanism for the development and maintenance of chronic pain complaints. However, another defensive response which is commonly studied in animals as a proxy for fear is freezing behavior. No research to date has investigated human freezing behavior in the context of pain. In addition, there is an increasing realization that social context can affect pain-relevant processes such as pain experience and pain behavior but less is known about the effects of social context on defensive responses to pain. Hence, this study investigated freezing behavior and facial pain expression in the context of pain, and their modulation by social context.

Methods. Healthy, pain-free participants $(\mathrm{N}=39)$ stood on a stabilometric force platform in a threatening or safe social context, which was manipulated using angry or happy facial stimuli. In some trials, an auditory cue (conditioned stimulus; CS) predicted the occurrence of painful electrocutaneous stimulus (unconditioned stimulus; pain-US). We assessed body sway (an index of freezing), heart rate, facial pain expression, self-reported pain intensity, unpleasantness, and pain-US expectancy during the CS and the context alone (no CS).

Results. The results were mixed. Neither the anticipation of pain, nor social context affected body sway. Heart rate and painful facial expression were reduced in the threatening social context at high anxiety levels. A threatening social context also elicited higher pain-US expectancy ratings. In sum, a threatening social context increases the expectation of pain, but reduces the facial expression of pain and lowers heart rate in highly anxious individuals. 
1

2

3

4

$5{ }^{1}$ Centre for the Psychology of Learning and Experimental Psychopathology, Faculty of

6 Psychology and Educational Sciences, KU Leuven, Leuven, Vlanders, Belgium

$7 \quad 2$ Research Group on Health Psychology, Faculty of Psychology and Educational Sciences, KU

8 Leuven, Leuven, Vlanders, Belgium

$9{ }^{3}$ Research Group on Experimental Health Psychology, Department for Clinical Psychological

10 Science, Faculty of Psychology and Neuroscience, Maastricht University, Maastricht, Limburg,

11 Netherlands

12

13

14

15

16

17

18

19

20

21 Correspondence concerning this article should be addressed to Dr. Kai Karos, Centre for the 22

Psychology of Learning and Psychopathology, KU Leuven, Tiensestraat 102, box 3726, 3000 
23 Leuven, Belgium. E-mail: Kai.Karos@kuleuven.be, T: +32 (0)16 3257 82, F: +32 (0)16 3261

2444.

25 Abstract

26 Background Maladaptive defensive responses such as excessive avoidance behavior have

27 received increasing attention as a main mechanism for the development and maintenance of

28 chronic pain complaints. However, another defensive response which is commonly studied in

29 animals as a proxy for fear is freezing behavior. No research to date has investigated human

30 freezing behavior in the context of pain. In addition, there is an increasing realization that social

31 context can affect pain-relevant processes such as pain experience and pain behavior but less is

32 known about the effects of social context on defensive responses to pain. Hence, this study

33 investigated freezing behavior and facial pain expression in the context of pain, and their

34 modulation by social context.

35 Methods Healthy, pain-free participants $(\mathrm{N}=39)$ stood on a stabilometric force platform in a

36 threatening or safe social context, which was manipulated using angry or happy facial stimuli. In

37 some trials, an auditory cue (conditioned stimulus; CS) predicted the occurrence of painful

38 electrocutaneous stimulus (unconditioned stimulus; pain-US). We assessed body sway (an index

39 of freezing), heart rate, facial pain expression, self-reported pain intensity, unpleasantness, and

40 pain-US expectancy during the CS and the context alone (no CS).

41 Results The results were mixed. Neither the anticipation of pain, nor social context affected

42 body sway. Heart rate and painful facial expression were reduced in the threatening social

43 context at high anxiety levels. A threatening social context also elicited higher pain-US 
44 expectancy ratings. In sum, a threatening social context increases the expectation of pain, but 45 reduces the facial expression of pain and lowers heart rate in highly anxious individuals.

\section{Introduction}

Pain is conceptualized and defined as a subjective response which is modulated by biological, psychological, and social factors [10,37]. While acute pain is adaptive in the shortterm and promotes healing, chronic pain persists beyond time of healing and is associated with severe disability and reduction in quality of life. Currently, chronic pain is a growing global health concern with 1 in 5 people in Europe and the US suffering from chronic pain $[8,41]$. Consequently, it is imperative to understand how chronic pain develops and is maintained. which proposes that pain-related fear and persistent maladaptive defensive responses such as avoidance lead to chronic pain-related disability. For example, patients might be afraid and consequently avoid activities that they believe to be associated with pain [48]. But how do formerly adaptive responses become maladaptive? Growing evidence suggests that the social context can play an important role. A threatening social context can worsen the experience of painful stimuli $[33,38,39]$, the communication of pain to others $[33,34,58]$, and facilitate the acquisition of pain-related fear [36]. More generally, chronic pain has been associated with a whole host of threatening social experiences such as social isolation and injustice [37]. In addition, early traumatic experiences of social threat such as bullying have been associated with increased risk for the development of chronic pain later on [18,72]. Similarly, people who are 
66

67

68

69

marginalized by social conditions (e.g., refugees, less well educated, living in poverty) are especially at risk [13].

Notwithstanding, the proposed importance of maladaptive defensive responses in the etiology and maintenance of chronic pain complaints in contemporary fear avoidance models, there is little research evaluating the modulation of defensive responses to pain by social context [38]. Most research to date has focused on behavioral avoidance responses, which are hypothesized to be at the center of the development and maintenance of chronic pain complaints $[48,73]$. However, avoidance is not the only defensive response of interest.

Another defensive response to threat, which is commonly used as the main outcome measure for fear in animal studies, is freezing. It is primarily characterized by reduced body motion and bradycardia (decreased heart rate) [20,22], but has also been associated with changes in the sympathetic nervous system (e.g., increased arterial pressure, increased respiration, increased muscle tone) [61]. While freezing has been studied in animal research for decades, research on human freeze-like behavior is only recently emerging [1,3,16,26,52]. Evidently, humans also show a freeze-like response (reduced body sway and bradycardia) when exposed to threatening films [23]. Especially relevant for the current study, another study demonstrated increased freeze-like behavior in humans in response to social threat (i.e., angry facial stimuli). Interestingly, this behavior was especially pronounced in highly anxious individuals [62]. This study was recently partly replicated, demonstrating that freezing occurs not only in response to physical threat but also social threat and is modulated by anxiety [56].

While adaptive in the short-term, freezing could become maladaptive when sustained and obstructing flexible adaptations to changes in the environment $[9,22,24]$. There is some evidence 
88 that increased freezing responses are associated with psychopathology, such as in the case of

89 aversive early life events [25], increased state anxiety [62], and increased nonspecific reduced

90 mobility in patients with panic disorders [46] but no such research exists within the domain of

91 pain. Similarly, to maladaptive avoidance behavior, prolonged freezing behavior could increase

92 the risk for the development of chronic pain (e.g., by facilitating physical immobility to

93 perceived threat of pain) and a threatening social context could be one of the factors facilitating

94 such a development.

Threat appraisal and associated defensive responses can directly affect pain reports $[29,30,69]$. However, research into human freezing responses in the context of pain is scarce. Animal research indicates that rats freeze during the anticipation of painful electrical stimuli $[12,63]$. Moreover, findings in human research are indicative of freezing responses to pain. A study in patients with chronic low back pain showed that patients exhibited freeze-like behaviors when instructed to move their trunk as fast as possible [6]. Another line of research demonstrated that pain behavior is reduced in a threatening social context. Peeters and Vlaeyen (2011) found that participants show less painful facial expression when receiving electrical stimuli in a threatening social context [58], possibly a result of freeze-like reductions of overall body movement. Subsequent attempts to replicate this finding have been mixed $[33,34]$. However, no study to date has investigated the effect of pain on actual body sway and bradycardia, or its modulation by social context. markers of freezing, specifically body sway, heart rate, and facial expressions of pain. Moreover, we investigated whether freezing responses were modulated by social context and anxiety levels. 
111 of pain. We hypothesized that the anticipation of pain would be associated with reduced body

112 sway (hypothesis 1), and that this effect would be more pronounced in a threatening social

113 context compared to a safe social context and in highly anxious individuals [62]. We also

114 hypothesized that a threatening social context overall is associated with bradycardia, especially

115 so in highly anxious individuals (hypothesis 2). In line with earlier research, we were also

116 interested whether painful facial expressions in the threatening social context are reduced

117 compared to a safe social context (hypothesis 3), which could be a sign of overall bodily

118 freezing.

\section{Materials and Methods}

\section{Participants}

Thirty-nine healthy, pain-free individuals (31 females; mean age $\pm \mathrm{SD}=22.79 \pm 3.07$; range $=18-33)$ voluntarily participated in this study. Participants were recruited by means of flyers and the departmental experiment management system (EMS; Sona Systems Ltd.). The majority of the participants were students $(n=34 ; 87 \%)$ and ten $(25.6 \%)$ participants were working. Of the 39 participants, 34 were living alone (87.2\%) and five (12.8\%) coinhabiting with someone else. Regarding highest education, 16 (41\%) participants had completed middle school,

$12710(25.6 \%)$ participants high school, and $13(33.3 \%)$ participants university education. The exclusion criteria were pregnancy, current or history of cardiovascular disease, chronic or acute respiratory disease (e.g., asthma, bronchitis), neurological diseases (e.g., epilepsy), any current or past psychiatric disorders, acute or chronic pain, uncorrected hearing problems, cardiac

131 pacemaker or the presence of any other electronic, medical devices, impaired, uncorrected

132 vision, or use of anxiolytics or antidepressants. All participants received a financial 
133 compensation of $€ 15$ for their participation. Power calculations were run using GPower [17],

134 assuming a medium effect size of Cohen's $\mathrm{f}=.25$, a (conservative) between-measurement

135 correlation of .05, an alpha level of .05, and aiming for a power of $90 \%$, resulting in a required

136 sample of 36 participants. A medium effect size was based on the range of effect sizes found in

137 the original study by Roelofs et al. [62].

\section{Ethical Approval}

The experimental protocol was approved by the Social and Societal Ethics Committee of the KU Leuven (Belgium) (registration number $=$ S-56678). All participants provided written informed consent prior to participation. It was emphasized that participation was completely voluntary and that participants were allowed to stop the experiment at any time without any negative consequences.

\section{Apparatus and experimental stimuli}

Stabilometric force platform. Postural stability and body sway of each participant were assessed using a NeuroCom Clinical Research System (NeuroCom International, Inc., Clackamas, OR, USA) (see http://www.interempresas.net/FotosArtProductos/P102631.jpg). The system comprises two independent $(23 \times 46 \mathrm{~cm}) 6$ degrees of freedom AMTI force plates and a three-sided surround. Vertical forces exerted on the plates were recorded at a sampling rate of $100 \mathrm{~Hz}$ and were used to derive the center of pressure (COP) time series for each participant in the anterior-posterior (AP), and medio-lateral (ML) directions. Participants wore a safety harness that was only engaged in the case of loss of balance. Note that the platform was stable at all times when participants stood on it. This relatively stable position enables a larger range of movement in the AP direction than in the ML direction, and therefore makes AP movements 
155 more susceptible than ML movements to affective modulations [62]. The platform also includes

156 a 15-inch, height-adjustable computer screen, which was positioned approximately $40 \mathrm{~cm}$ in

157 front of the participant adjusted to eye-level.

158 Heart rate monitor. Heart rate was measured as beats per minute (bpm) with a Polar

159 heart rate monitor (Polar Electro Oy, Kempele, Finland), which consists of an electrode belt and

160 transmitter W.I.N.D and a wireless heart rate monitor RS800CX.

161 Software. The entire experiment was run on a Windows XP computer (Dell Optiplex

162 755) with 2 GB RAM and an Intel Core 2 Duo processor at $2.33 \mathrm{GHz}$ and an ATI Radeon 2400

163 graphics card with 256 MB of video RAM. Stimulus presentation was controlled by Affect

164 (version 4.0) [68].

165 Stimulus material. A painful electrocutaneous stimulus of $2 \mathrm{~ms}$ duration (single squared 166 waveform pulse) with a $2000 \mu$ s pulse duration and a maximum voltage of $400 \mathrm{~V}$ served as the 167 unconditioned stimulus (pain-US) in the present experiment. The electrical stimulation was 168 delivered by a commercial stimulator (DS7A, Digitimer, Welwyn Garden City, England) through 169 surface SensorMedics electrodes (1 cm diameter) filled with K-Y gel that were attached to the 170 wrist of the right hand of the participant. To select the intensity level of the pain-US, participants

171 were repeatedly exposed to electrocutaneous stimulation of increasing intensity. They were

172 asked to rate each stimulus on a scale ranging from 0 (feeling nothing) to 10 (worst pain 173 imaginable). The participant was instructed to select a stimulus intensity with a rating of about 8 ,

174 which was "moderately painful and demanding some effort to tolerate" (mean self-reported

175 stimulus intensity was $8.08, S D=0.27$, range $=8-9$ ). After selecting the pain stimulus the

176 participant was informed that (s)he would repeatedly receive stimuli of the maximum calibrated

177 intensity during the remainder of the experiment. They were also given the possibility to increase 
178 or decrease the selected stimulus intensity at this point (mean physical stimulus intensity was

$17932.75 \mathrm{~mA}, S D=20.55$, range $=2-84 \mathrm{~mA})$.

Based on previous a previous pilot test, a $44100 \mathrm{~Hz}$ auditory cue of $1 \mathrm{~s}$ duration presented

181

182

183

184

185

186

187

binaurally via headphones at 90 dBA (HD 202, Sennheiser) served as the conditioned stimulus (CS). Social context was manipulated using facial stimuli. The facial stimuli consisted of emotional faces taken from 20 models (10 male and 10 female) in the Karolinska Directed Emotional Faces database [47]. Each model showed two different affective expressions (happy and angry), resulting in a total of 40 stimuli. The stimuli were the same as used in the study by Roelofs, Hagenaars and Stins [62].

\section{Experimental setting}

The experiment took place in a dimmed experimental room. During the experiment participants stood on the stabilometric force platform for the majority of the time while the experimenter was present in the same room but out of sight.

\section{Procedure}

The experimental session lasted approximately 120 minutes and consisted of a preparation and calibration phase, an acquisition phase, and a generalization phase. Figure 1 provides a more detailed overview of the acquisition and generalization phases, which we subsequently refer to as the experimental phases. A within-subject design was employed, meaning that all participants ran through both threatening and safe contexts. Note that the generalization phase was exploratory. Considering the high drop out in this phase the findings are unreliable and thus should be interpreted with caution. We decided to include a full 
199 description of the methods and results of the generalization phase in the supplementary material

200 only rather than in the main manuscript to facilitate clarity (see Supplementary material 1).

Preparation and calibration phase. After participants arrived at the lab they were

203

204

205

206

207

208

209

210

211

212

213

214

215

216

217

informed about the study orally and in writing. The participants were led to believe that the study concerned the effects of different kinds of distractors (auditory, visual and sensory) on balance (i.e., standing still on the platform). They were informed that painful electrocutaneous stimuli (pain-USs) would be administered during the experiment. After the participants provided informed consent, the equipment for the heart rate measurement was attached. Subsequently, the calibration of the electrocutaneous stimulus was performed. Afterwards the use of the platform was explained, and participants removed their shoes and attached the safety harness. They were instructed to stand centrally on the platform with their arms hanging alongside their body, to move as little as possible during the experiment, and to watch the computer screen in front of them. Lastly, the electrodes for the pain-US administration were reattached and the safety harness was connected to the platform. Participants then read the following task instructions on the computer screen: "Please stand still on the platform and try to move as little as possible. You will be presented with several stimuli. Please focus on the center of the screen during the whole experiment. Sometimes you will be presented with sounds. These sounds might be followed by an electrocutaneous stimulus."

and 3 blocks with happy facial stimuli). Henceforth, these blocks will be referred to as A1 to A3 (also see Figure 1). The order of the block presentation was intermixed, and the order of stimuli 
221 within each block was randomized. Each block consisted of 20 images of one type of emotional

222 expression. During each trial the facial stimulus was presented for $3 \mathrm{~s}$. In $50 \%$ of the trials (10

223 per block) an auditory cue was presented $1.5 \mathrm{~s}$ after the start of the trial (CS + trials). In half of

224 these trials ( 5 per block), the auditory cue would be followed by an electrocutaneous stimulus 1.3

225 s after the auditory cue (i.e., 50\% reinforcement rate). The five trials with electrocutaneous

226 stimuli would last for $5 \mathrm{~s}$ instead of $3 \mathrm{~s}$ to allow time for recovery. In the remaining $50 \%$ of trials

227 (10 per block), no auditory cue or electrocutaneous stimulus was presented (context-alone trials).

228 Thus, each block would take $70 \mathrm{~s}$ in total to complete.

After each block the safety harness was unhooked and the electrodes disconnected. The

participant stepped off the platform and sat down at the table to fill in several questionnaires (see

Outcome measures). This break would take approximately 5 minutes between blocks.

232 Afterwards the same procedure was repeated for the remaining blocks.

\section{Outcome measures}

\section{Self-report measures}

235

Pain-US expectancy. In order to assess whether differential learning occurred and as a

proxy for pain-related fear [5], pain-US expectancy ratings were collected. Participants were

presented with the following questions: (1) "During the last block, how much did you expect that a face together with a tone would be followed by an electrical stimulus?" and (2) "During the last block, how much did you expect that a face without a tone would be followed by an electrical

241 at all" to "very much". 
were asked the following questions: (1) "How painful did you find the painful stimuli in the last

244

block?" and, (2) "How unpleasant did you find the painful stimuli in the last block?" which they rated on an 11-point Likert scale ranging from 0 to 10. The anchors were (1) "not painful at all" and "very painful" and (2) "not unpleasant at all" and (10) "very unpleasant".

\section{Retrospective affective valence, arousal and control of the facial stimuli and}

auditory cue. After each block, participants rated valence, arousal and sense of control of the facial stimuli using the Self-Assessment Manikin scale (SAM) [7] consisting of 5 pictographs.

Participants retrospectively rated how they felt when exposed to the facial stimuli. All responses were scored from 1 (very unhappy/not at all aroused/no control) to 5 (very happy/very aroused/full control). Similarly, at the beginning of the experiment (before the first block), and after the last block participants rated the auditory cue on these three measures.

Perceived threat and pleasantness of facial stimuli. After each block, participants answered the following questions: 1) "How threatening did you find the facial stimuli in the last block?" and 2) "How pleasant did you find the facial stimuli in the last block?" on an 11-point Likert scale ranging from 0 (not threatening at all/not pleasant at all) to 10 (very threatening/very pleasant). These measures were included as a manipulation check for the social context manipulation.

State-trait anxiety inventory. As in the study by Roelofs, Hagenaars and Stins [62], trait anxiety was measured by the trait version of the Spielberger's State-Trait Anxiety Inventory (STAI-T). This questionnaire assesses how anxious participants feel in general, using a scale ranging from 1 (not at all) to 4 (very much) (e.g., "I feel nervous and restless."), and the scale 
265 has been shown to have high internal consistency, satisfactory test-retest reliability and

266 concurrent validity with other anxiety measures [67]. Mean anxiety scores in our sample were

$26737.61(\mathrm{SD}=9.68$, range $=24-66)$.

\section{Behavioral outcomes}

Body sway. Body sway was measured as an index of postural mobility [40,62]. For each trial, the mean position of the center of pressure (COP) in the anterior-posterior (AP) and the medio-lateral (ML) directions was calculated. Referencing this mean, the standard deviation of the COP in the AP direction was computed and used as an index of variability in body sway. The standard deviations were then averaged across each block and stimulus type (CS/context-alone), excluding all trials where the pain-US was administered ( $25 \%$ of trials). In line with several earlier studies, we focused on variations in the AP-direction in our analyses, as these are more susceptible to affective modulations $[23,54,55,62]$. to capture participants' facial expression. The webcam was installed right above the stimulus screen and adjusted per participant to capture the whole face during the experiment. Video tapes of each participant were rated using the Facial Action Coding System (FACS) [15], a finegrained anatomically based system that is considered the criterion standard when decoding facial expressions, including the facial expression of pain [59]. Six facial action units which have been found to most reliably indicate pain are brow lower, eye squeeze, eye squint, nose wrinkle, check raiser and upper lip raise $[42,58-60]$ were rated by the third author (TL), who was trained by the first author. The first author (KK), who is a certified FACS coder, also independently rated a randomly selected $20 \%$ subset of all video fragments. Each video fragment consisted of four- 
287 second segments capturing one second prior to, and three seconds after administration of the

288 pain-US. Each second of the four-second interval was coded using a software program enabling

289 the rater to view and review each second at normal rate and at a rate of one-tenth of a second.

290 For each time interval, a mean score per second for each of the six facial actions was calculated.

291 A total score was calculated by summing these mean scores per participant [11]. Reliability was

292 calculated according to the formula given by Ekman and Friesen [15] which assesses the

293 proportion of agreement on actions recorded by two coders relative to the total number of actions

294 coded as occurring by each coder. Inter-rater reliability was satisfactory and ranged from .89 to

295.95 across all facial actions.

Heart rate. The Polar belt and transmitter supported recording and processing of R-R

297

298

299

300

301

302

303

304

305

306

307

intervals at a frequency of $1000 \mathrm{~Hz}$ and $2.4 \mathrm{GHz}$ transfer between the belt and heart rate monitor.

The portable belt was attached around the chest at the height of the sternum. Unfortunately, the

Polar did not make it possible to distinguish between individual trials so heart rate responses were averaged across blocks.

\section{Statistical Analyses and Data Reduction}

Paired-samples t-tests were carried out to compare the level of arousal, control and valence after hearing the auditory cue (CS) before and after it was paired with the painful stimulus. As a manipulation check for the social context manipulation, paired-samples t-tests were conducted to compare the valence, arousal, control, threat, and pleasantness ratings in response to the happy and angry facial stimuli. To this end, all ratings were averaged separately for the safe and threatening blocks.

Pain-US expectancy ratings and body sway were analyzed using repeated measures (RM) ANOVAs with 2 [Stimulus type (CS+/context-alone)] x 2 [Context (threat/safe)] x 3 [Block (A1, 
310 A2, A3)] as within-subject factors. Separate 2 [Context (threat/safe)] x 3 [Block (A1, A2, A3)]

311 RM ANOVAs were run to examine the effects of social context on heart rate, facial pain

312 expression and pain ratings (pain intensity and pain unpleasantness). In the analyses of heart rate,

313 painful facial expression and body sway, centered STAI-T scores were included as a covariate.

314 To deal with frequent violations of the assumption of sphericity, multivariate analyses

315 were run. Pillai's trace estimates and the effect size indication $\eta_{p}^{2}$ are reported. Planned

316 comparisons were carried out to test our a priori hypotheses. Holm Bonferroni corrections were

317 used to correct for multiple testing [28]. All statistical analyses were run using IBM SPSS 20

318 (SPSS Inc, Chicago, IL). An alpha level of .05 was used for all statistical tests. We report how

319 we determined our sample size, all data exclusions (if any), all manipulations, and all measures

320 in the study [66].

321

\section{Results}

\section{Dropout}

The experimental procedure was quite demanding for the participants and 6 participants stopped their participation before the end of the experiment because they indicated that they started to feel unwell and as a precaution the experimenter decided to terminate the experiment.

326 From the initial 39 participants, 33 participants $(84.6 \%)$ completed the full experiment including

327 the generalization phase, and 36 participants (92.3\%) completed only the acquisition phase

328 which is reported in this manuscript. The three (7.7\%) remaining participants stopped their 329 participation during the acquisition blocks. All subsequent analyses are run on the 36 participants 330 who completed the acquisition phase unless otherwise stated.

\section{Manipulation checks}


no significant difference in reported level of arousal, Mbefore $=2.50, S D=.97 ;$ Mafter $=2.42, S D=$

334

335

336

337

338

339

340

341

342

343

344

345

346

347

$.10 ; t(35)=.400, p=.69$, and only a slight trend with regard to valence, Mbefore $=2.86, S D=.97$;

$\mathrm{M}_{\mathrm{after}}=2.61, S D=.76 ; t(35)=1.86, p=.07$, after hearing the auditory cue $(\mathrm{CS})$ before and after

the experiment. However, the reported level of control after hearing the auditory cue (CS) before and after the experiment significantly decreased, Mbefore $=4.14, S D=1.40$; Mafter $=3.61, S D=$ $1.40 ; t(35)=2.08, p=.05$.

\section{Retrospective perceived threat, pleasantness, affective valence, arousal and control}

of the facial stimuli. As anticipated, angry faces were rated as less pleasant, $\mathrm{Mangry}=2.82, \mathrm{SD}=$ $1.45 ;$ Mhappy $=6.02, \mathrm{SD}=1.88, t(35)=-7.76, p<.01$, and more threatening, $\mathrm{M}_{\text {angry }}=5.81, \mathrm{SD}=$ 1.69; $\mathrm{M}_{\text {happy }}=2.36, \mathrm{SD}=2.01, t(35)=9.05, p<.01$, than happy faces. Furthermore, participants indicated that they felt more unhappy (lower scores indicate more unhappiness), Mangry $=2.28$, $\mathrm{SD}=.57 ;$ Mhappy $=3.31, \mathrm{SD}=.76 ; t(35)=-7.74, p>.01$, while looking at the angry faces and also rated them as more arousing, Mangry $=2.96, \mathrm{SD}=.73 ;$ Mhappy $=2.57, \mathrm{SD}=.72 ; t(35)=3.93$, $p<.01$, than happy faces. There was no significant difference in reported level of control, Mangry $=2.53, \mathrm{SD}=.84 ;$ Mhappy $=2.65, \mathrm{SD}=.78 ; t(35)=-1.10, p=.28$, between angry and happy faces. These results indicate that the social context manipulation was successful.

\section{Pain-US expectancy ratings}

We found support for differential acquisition: Participants increasingly expected the painUS following the presentation of the CS, but not when the context was presented alone, Stimulus type $\mathrm{x}$ Block, $F(2,34)=8.79, p<.01, \eta_{p}^{2}=.34$, (see Figure 2 ). However, the magnitude of the differential acquisition effect did not differ between the two contexts, Stimulus type x Block x 
354 Context, $F(2,34)=1.17, p=.32, \eta_{p}^{2}=.07$. In contrast, pain-US expectancy ratings were overall

355 higher in the threatening context, $M_{\text {threat }}=6.70, S D_{\text {threat }}=1.85$, compared to the safe context,

$356 M_{\mathrm{safe}}=6.01, S D_{\mathrm{safe}}=.2 .21$, main effect of Context: $F(1,35)=5.87, p=.02, \eta_{p}^{2}=.14$.

357

358

359

360

361

362

363

364

365

366

367

368

369

370

371

372

\section{---- INSERT FIGURE 2 ABOUT HERE ---}

\section{Pain intensity and unpleasantness ratings}

Pain intensity ratings did not differ across blocks, main effect of Block: $F(2,34)<1, p=$ $.64, \eta_{p}^{2}=.03$, or between contexts, main effect of Context: $F(1,35)<1, p=.68, \eta_{p}^{2}<.01$, and there was also no interaction between block and context, Block $\mathrm{x}$ Context interaction: $F(2,34)=$ $1.03, p=.37, \eta_{p}^{2}=.06$. The same was true for pain unpleasantness ratings, Block: $F(2,34)<1, p$ $=.42, \eta_{p}^{2}=.05$; Context: $F(1,35)<1, p=.56, \eta_{p}^{2}=.01$, Block x Context interaction: $F(2,34)<$ $1, p=.96, \eta_{p}^{2}<.01$

\section{Hypothesis 1: Does social threat modulate body sway in response to pain?}

Two participants were identified as outliers because of their excessive movements, as reflected in Z-scores greater than 4 on the body sway measures ${ }^{1}$. Consequently, we decided to remove these participants from the analyses. Thus, the following analyses were run on 34 participants. The four-way interaction was not significant, Context x Block x Stimulus x Trait anxiety, $F(2,30)<1, p=.74, \eta_{p}^{2}=.02$, and neither were any of the other interaction or main effects. There was a slight trend showing less body sway during CS trials compared to context-

\footnotetext{
${ }^{1}$ The results of these analyses remained the same even when the outliers were included.
} 
373 alone, Stimulus, $F(1,31)=3.03, p=.09, \eta_{p}^{2}=.09$, but contrary to our hypothesis we found no

374 evidence for any effect of social context or painful stimuli on body sway.

375 Hypothesis 2: Does social threat reduce heart rate?

Because of technical difficulties with the heart-rate monitor, recordings of three

377 participants were missing and hence the following analyses are run on 33 participants. With

378 regard to heart rate, a significant three-way interaction emerged, Block x Context x Trait anxiety,

$379 F(2,30)=3.68, p=.04, \eta_{p}^{2}=.20$ (see Figure 3$)$. We then evaluated context differences per block

380 for high (mean STAI-T + 1SD) and low levels of trait anxiety (mean STAI-T - 1SD) using

381 planned comparisons. Note that this analysis models the interaction of block and context at

382 different levels of the covariate ( +1 SD and -1 SD) and thereby avoids the power loss associated

383 with a median split. The model is thus based on the whole sample [see 27 for use of a similar

384 analysis].

385 While there were no differences between a threatening and a safe social context in any of

386 the blocks at low anxiety levels, $F(1,31)<1, p=.89, \eta_{p}^{2}<.01$, there was a difference in heart

387

388

389

390

391

392

393

394

395

rate at high anxiety levels. Specifically, the threatening social context, $M_{\text {threat }}=645.47, S D_{\text {threat }}=$ 141.49 , led to reduced heart rate in the very last block compared to a safe social context, $M_{\text {safe }}=$ 659.49, $S D_{\text {safe }}=147.64, F(1,31)=9.92, p<.01, \eta_{p}^{2}=.24$

\section{--- INSERT FIGURE 3 ABOUT HERE ---}

\section{Hypothesis 3: Does social threat reduce facial pain expression?}

Because of technical difficulties recordings of three participants were missing and hence the following analyses are run on 33 participants. Regarding facial pain expression, no significant three-way interaction emerged, Block $\mathrm{x}$ Context $\mathrm{x}$ Trait anxiety, $F(2,30)=1.26, p=$ 
$396.30, \eta_{p}^{2}=.08$. As expected, we found a significant effect of social context that was moderated by

397 trait anxiety, Context $x$ Trait anxiety, $F(1,31)=5.35, p=.03, \eta_{p}^{2}=.15$, (see Figure 4).

398 Subsequently, we evaluated simple effects of social context at high anxiety levels (1 SD above 399 mean STAI-T score) and low anxiety levels (1 SD below mean STAI-T score). In line with our 400 hypotheses, we found that there is less facial pain expressions in the threatening context at high 401 anxiety levels, $M_{\text {threat }}=2.62, S D_{\text {threat }}=5.27$, compared to the safe context, $M_{\text {safe }}=3.55, S D_{\text {safe }}=$

$4025.56, F(1,31)=4.89, p=.04, \eta_{p}^{2}=.14$. In contrast, there was no difference between the two 403 contexts at low anxiety levels, $M_{\text {threat }}=3.56, S D_{\text {threat }}=4.91 ; M_{\text {safe }}=3.09, S D_{\text {safe }}=5.21, F(1,31)=$ 404 $1.24, p=.27, \eta_{p}^{2}=.04$

405

406 --- INSERT FIGURE 4 ABOUT HERE---

407

408

\section{Discussion}

The current study aimed to explore the effects of a threatening versus a safe social context on freezing responses to pain, hypothesizing that (1) the anticipation of pain is associated with reduced body sway, and that this response is more pronounced in a threatening social context compared to a safe social context and especially so for highly anxious individuals, (2) that a threatening social context would induce bradycardia, and (3), reduce painful facial expression compared to a safe social context.

416 (hypothesis 1), or that body sway is modulated by social context. That is, we were not able to 417 replicate the results reported by Roelofs, Hagenaars and Stins [62], who demonstrated that angry 
418 facial stimuli lead to reductions in body sway compared to happy facial stimuli. In addition, even

419 though animal research indicates that the anticipation of painful electrocutaneous stimuli leads to

420 freezing in rats [12], we did not see reductions in body sway in the anticipation of painful stimuli

421 in humans. A possible explanation for this finding might relate to the proposed evolutionary

422 function of freezing behavior. There is increasing evidence that freezing is an active preparation

423 response rather than a passive orientation response [19,22]. That is, even though the present

424 study and several earlier studies investigated freezing using passive viewing paradigms, one

425 might expect freezing responses especially in situations where action is possible to respond to

426 threat (e.g., a situation where one could avoid threat) rather than in a situation of helplessness as

427 was the case here. A recent study by Gladwin, Hashemi, van Ast and Roelofs [19] indeed found

428 that freezing in the form of reduced body sway and bradycardia was strongly related to the

429 possibility to respond. That is, freezing was shown during a preparatory period in a virtual

430 shooting simulation when participants had the possibility to respond to a possible attack in

431 comparison to a situation where they did not have that possibility. Another possible explanation

432 for this finding is that painful electrocutaneous stimuli themselves had a substantial effect on

433 body sway in this study, overshadowing possible effects of social context or pain anticipation on

434 body sway. Since we administered electrocutaneous stimuli in both contexts, we might have

435 induced overarching contextual threat that led to freezing independent of social context. In other

436 words, we did not create a truly safe context as a comparison. The addition of a baseline

437 condition without painful stimuli might solve this problem. Another possible explanation might

438 be that in contrast to the study by Roeloefs, Hagenaars, and Stins [62], all participants wore a

439 safety harness during the study which might have affected mobility itself or acted as a safety

440 signal which reduced overall threat perceptions during the study. 
442 to reductions in heart rate (bradycardia) compared to a safe social context, we only found a

443 highly specific effect: Social threat led to bradycardia at high anxiety levels and only so in the

444 last block. So while these findings partly replicate the findings by Roelofs, Hagenaars and Stins

445 [62], the effect of social threat seems to be rather specific for highly anxious individuals. We also

446 observed an overall decline in heart rate across blocks at high anxiety levels (independent of

447 social context) but not at low anxiety levels which is in line with earlier research in rodents [22],

448 and a similar time-course has been demonstrated in response to affective films [23]. This long-

449 lasting time course demonstrates that the current paradigm might indeed cause freezing, a

450 sustained defensive response, rather than just short-lasting orienting responses. In sum, as

451 freezing in humans and animals is commonly defined as a reduction in mobility and bradycardia

$452[22,23,62]$, we can conclude that we found no evidence for freezing in the current study.

Third, mirroring the heart rate findings, we also found an effect of social threat on pain

expression (hypothesis 3), but again limited to high anxiety levels. A threatening social context

led to reduced facial pain expression in comparison to the safe social context at high anxiety

levels. These findings support an evolutionary account proposed by Williams [74], which

457 proposes that it might be disadvantageous to express pain in a threatening social environment as

458 it could indicate vulnerability which might be exploited by adversaries. Thus far, three empirical

459 studies have supported this account $[33,58,75]$ and the current study also supports this idea,

460 except that this effect was limited to highly anxious individuals.

462 reflect conscious control or automatic processes (or both). While it has been argued that facial 
464 present findings might lend some support for automaticity as well. While an earlier study found 465 inhibition of facial pain expression in response to real-life others [58], we demonstrated this 466 inhibition for the first time in response to inanimate facial stimuli. One possible explanation is 467 that these stimuli indeed acted as social threat cues and activated hard-wired, automatic reactions 468 in facial pain display as proposed by evolutionary accounts of pain expression [74]. It is also 469 interesting to note, that this inhibition was only observed in highly anxious individuals. That is, 470 highly anxious individuals were more sensitive to a threatening social context than low anxious 471 individuals and consequently, demonstrated defensive responding in response to passive facial 472 stimuli. This finding is rather surprising, considering that anxiety has been associated previously 473 with a lack of inhibitory capacity [2]. This finding may be clinically relevant, as patients with 474 pain complaints might inhibit facial expression of pain if they do not feel safe (e.g., do not trust a 475 healthcare professional). One possible consequence of this might be that the pain of the patient is 476 underestimated by others, as is commonly the case in clinical practice $[33,64]$. freezing response (i.e., reductions in body sway and bradycardia), suggesting that these are likely independent constructs. While reductions in facial pain expression could be explained by general body immobility as demonstrated in the context of freezing, the current study demonstrates that inhibition of facial pain expression can occur independently of observed freezing behavior.

Fourth, we also explored the effects of a threatening social context on pain expectancy, pain intensity and pain unpleasantness. We found no support that social threat directly affects pain intensity or unpleasantness ratings. There is limited support that a threatening social context in the form of intentional pain by others might directly affect pain intensity ratings $[21,33,39]$ but 
487 using angry and happy facial stimuli to manipulate social context has found that fear learning

488 was facilitated in a threatening social context [36]. In the present study, we found that overall

489 pain expectancy ratings were higher in the threatening social context compared to the safe social

490 context. That is, participants expected more pain in the threat context independently of the

491 presence (or absence) of the CS. This finding seems to demonstrate contextual fear (i.e.,

492 hypervigilance), which is usually observed in a context where cued learning is impossible (e.g.,

493 an unpredictable US) [50], or when individuals fail to learn the CS-US association [4]. In the

494 current study, participants expected more pain in the threatening social context despite learning

495 the CS-US association. In other words, a threatening social context led participants to predict that

496 the occurrence of threatening stimuli (i.e., pain) would be more likely.

There are a few limitations to this study that should be noted. First, for practical reasons

we were only able to measure heart rate across blocks rather than per trial, which would have

been desirable to distinguish between pain and non-pain trials. This would allow a direct comparison with the body sway data, which could be evaluated on a trial-by-trial basis. Second, the current study suffered from drop out which means that some of the analyses (specifically, the heart-rate and pain expression analyses) are not as robustly powered as we would have hoped 503 and that the results presented here should be interpreted with caution. It should be noted that the participants who completed the full experiment and those who dropped out before the end did not differ in regard to anxiety, pain catastrophizing, fear of pain, or gender. Third, there are several variables that might affect postural stability which were not recorded in the present study

507 (e.g., physical activity levels, sleep, use of drugs, alcohol, or nicotine). Some of these variables might have overshadowed the manipulation used in this study and should therefore be assessed and controlled for in future studies. Fourth, we found large variability in heart-rate and facial 
510 pain expression scores which suggests for large inter-individual variability. Even though this is

511 in line with earlier studies $[33,65]$, future studies might attempt to control for confounding

512 variables (e.g., physical activity, gender, age) to reduce this variability and increase statistical

513 power. Fifth, the current study recruited pain-free controls and made use of an experimental pain

514 induction method. Consequently, any extrapolations of the findings to patients with chronic pain

515 are premature at this stage. However, the basic assumption with this fundamental research is that

516 the underlying mechanisms in healthy populations under certain manipulations can inform us

517 about what is happening in patient populations. Lastly, the current study did not have a

518 predefined and publicly available analysis plan prior to the start of the study. Even though this

519 was not common practice at the time that this study was conducted, future studies should

520 preregister their plan of analysis and hypotheses to further limit potential bias and facilitate

521 transparency in science $[45,57]$.

\section{Conclusions and future directions}

In conclusion, the current study provides preliminary evidence that social context modulates pain-relevant processes, especially in highly anxious individuals. Social threat increased overall expectation of pain and reduced facial pain expression. In addition, increased bradycardia in the threatening social context was restricted to a single block and only present in highly anxious participants. In contrast, we did not find freezing in response to painful stimuli and also no effect of social context on body sway. This research relates to earlier studies showing

529 impaired safety learning and excessive avoidance behavior in highly anxious individuals, and 530 might add to our understanding of how chronic pain complaints develop and are maintained 531 [49,51]. In addition, the present research further highlights the importance of social context in 532 the study and understanding of pain [38]. 
534 the effects of acute and chronic pain on freezing parameters. To this end, the effect of

535 experimental pain induction methods and anticipation of pain on freezing responses in humans

536 should be investigated in isolation. In addition, freezing responses in patients with chronic pain

537 should be compared to pain-free controls to investigate possible differences in defensive

538 responding. There is some evidence for maladaptive freezing responses in other

539 psychopathologies such as PTSD [53] and in rats with chronic pain [44], but research in humans

540 is absent. It is well established that patients move differently when they are in pain [27] and even

541 the anticipation of pain changes movement [32]. If chronic pain patients do show signs of

542 maladaptive freezing responses, as they do with avoidance behavior, longitudinal research is

543 needed to evaluate the role of this process in the etiology of chronic pain complaints. Second,

544 more research into the modulation of defensive behaviors (e.g., freezing, pain-related avoidance

545 behavior) by social context is warranted. One possibility is the use of more dynamic and

546 ecologically valid social manipulations such as virtual reality, video materials or actual social

547 interactions using confederates [33,35].

\section{5. Acknowledgements}

549 The authors thank Jeroen Clarysse, Mathijs Franssen and Professor Ralf Krampe for their 550 technical support.

\section{6. References}

553 [1] Allcoat D, Greville WJ, Newton PM, Dymond S. Frozen with fear: Conditioned suppression in a virtual reality model of human anxiety. Behav Processes 2015;118:98-

555 101. doi:10.1016/j.beproc.2015.06.011. 
556 [2] Ansari TL, Derakshan N. The neural correlates of impaired inhibitory control in anxiety.

557 Neuropsychologia 2011;49:1146-1153. doi:10.1016/j.neuropsychologia.2011.01.019.

558 [3] Azevedo TM, Volchan E, Imbiriba LA, Rodrigues EC, Oliveira JM, Oliveira LF,

559 Lutterbach LG, Vargas CD. A freezing-like posture to pictures of mutilation.

$560 \quad$ Psychophysiology 2005;42:255-260.

561 [4] Baas JMPMP, van Ooijen L, Goudriaan A, Kenemans JLL. Failure to condition to a cue is

$562 \quad$ associated with sustained contextual fear. Acta Psychol (Amst) 2008;127:581-592.

563 doi:10.1016/j.actpsy.2007.09.009.

564 [5] Boddez Y, Baeyens F, Luyten L, Vansteenwegen D, Hermans D, Beckers T. Rating data 565 are underrated: validity of US expectancy in human fear conditioning. J Behav Ther Exp 566 Psychiatry 2013;44:201-6. doi:10.1016/j.jbtep.2012.08.003.

567 [6] Bourigua I, Simoneau EM, Leteneur S, Gillet C, Ido G, Barbier F. Chronic low back pain 568 sufferers exhibit freezing-like behaviors when asked to move their trunk as fast as $569 \quad$ possible. Spine J 2014;14:1291-1299.

570 [7] Bradley MM, Lang PJ. Measuring emotion: The self-assessment manikin and the semantic differential. J Behav Ther Exp Psychiatry 1994;25:49-59. doi:10.1016/0005-

572 7916(94)90063-9.

573 [8] Breivik H, Collett B, Ventafridda V, Cohen R, Gallacher D. Survey of chronic pain in $574 \quad$ Europe: Prevalence, impact on daily life, and treatment. Eur J Pain 2006;10:287-287. doi:10.1016/j.ejpain.2005.06.009.

576 [9] Buss K a, Davidson RJ, Kalin NH, Goldsmith HH. Context-specific freezing and 577 associated physiological reactivity as a dysregulated fear response. Dev Psychol 
579 [10] de C Williams AC, Craig KD, Williams ACDC, Craig KD. Updating the definition of pain. Pain 2016;157:2420-2423. doi:10.1097/j.pain.0000000000000613.

581 [11] Caes L, Vervoort T, Trost Z, Goubert L. Impact of parental catastrophizing and contextual threat on parents' emotional and behavioral responses to their child's pain. Pain 2012;153:687-695. doi:10.1016/j.pain.2011.12.007.

584

585

586

587

588

589

590

591

592

593

594

595

596

597

598

599

[12] de Castro Gomes V, Landeira-Fernandez J. Amygdaloid lesions produced similar contextual fear conditioning disruption in the Carioca high- and low-conditioned freezing rats. Brain Res 2008;1233:137-145. doi:10.1016/j.brainres.2008.07.044.

[13] Craig KD, Holmes C, Hudspith M, Moor G, Moosa-Mitha M, Varcoe C, Wallace B. Pain in persons who are marginalized by social conditions. Pain 2020;161:261-265.

[14] Crombez G, Eccleston C, Van Damme S, Vlaeyen JWSS, Karoly P. Fear-avoidance model of chronic pain: the next generation. Clin J Pain 2012;28:475-83. doi:10.1097/AJP.0b013e3182385392.

[15] Ekman P, Friesen W V. Facial action coding system. Palo Alto: Palo Alto : Consulting psychologists, $1978 \mathrm{p}$.

[16] Facchinetti LD, Imbiriba LA, Azevedo TM, Vargas CD, Volchan E. Postural modulation induced by pictures depicting prosocial or dangerous contexts. Neurosci Lett 2006;410:52-56.

[17] Faul F, Erdfelder E, Lang A-GGA-G, Buchner A, Kiel C. G*Power 3: A flexible statistical power analysis program for the social, behavioral, and biomedical sciences. Behav Res Methods 2007;39:175-191. doi:10.3758/bf03193146. 
600 [18] Fekkes M, Pijpers FIM, Fredriks AM, Vogels T, Verloove-Vanhorick SP. Do bullied

601 children get ill, or do ill children get bullied? A prospective cohort study on the

602 relationship between bullying and health-related symptoms. Pediatrics 2006;117:1568-74.

603 doi:10.1542/peds.2005-0187.

604 [19] Gladwin TE, Hashemi MM, van Ast V, Roelofs K. Ready and waiting: Freezing as active 605 action preparation under threat. Neurosci Lett 2016;619:182-188.

606 doi:10.1016/j.neulet.2016.03.027.

607 [20] Glombiewski JA, Riecke J, Holzapfel S, Rief W, König S, Lachnit H, Seifart U. Do

608 patients with chronic pain show autonomic arousal when confronted with feared

609 movements? An experimental investigation of the fear-avoidance model. Pain

$610 \quad 2015 ; 156: 547-554$.

611 [21] Gray K, Wegner DM. The sting of intentional pain. Psychol Sci 2008;19:1260-2.

612 doi:10.1111/j.1467-9280.2008.02208.x.

613 [22] Hagenaars M a, Oitzl M, Roelofs K. Updating freeze: Aligning animal and human 614 research. Neurosci Biobehav Rev 2014;47C:165-176.

615 doi:10.1016/j.neubiorev.2014.07.021.

616 [23] Hagenaars M a, Roelofs K, Stins JF. Human freezing in response to affective films.

617 Anxiety Stress Coping 2014;27:27-37. doi:10.1080/10615806.2013.809420.

618 [24] Hagenaars M, van Minnen a, Holmes E, Brewin C, Hoogduin K. The effect of 619 hypnotically induced somatoform dissociation on the development of intrusions after an

620 aversive film. 2008:37-41. doi:10.1080/02699930701575151.

621 [25] Hagenaars MA, Stins JF, Roelofs K. Aversive life events enhance human freezing 
622

623 [26] Hermans EJ, Henckens MJ a G, Roelofs K, Fernández G. Fear bradycardia and activation 624

625

626

627

628

629

630

631

632

633

634

635

636

637

638

639

640

641

642

643

responses. J Exp Psychol Gen 2012;141:98-105.

of the human periaqueductal grey. Neuroimage 2013;66:278-287.

doi:10.1016/j.neuroimage.2012.10.063.

[27] Hodges PW, Tucker K. Moving differently in pain: A new theory to explain the adaptation to pain. Pain 2011;152:S90-S98. doi:10.1016/j.pain.2010.10.020.

[28] Holm S. A Simple Sequentially Rejective Multiple Test Procedure. Scand J Stat 1979;6:65-70.

[29] Jackson T, Huang X, Chen H, Phillips H. Effects of threatening information on interpersonal responses to pain. Eur J Pain 2009;13:431-8.

doi:10.1016/j.ejpain.2008.05.012.

[30] Jackson T, Pope L, Nagasaka T, Fritch A, Iezzi T, Chen H. The impact of threatening information about pain on coping and pain tolerance. Br J Health Psychol 2005;10:44151. doi:10.1348/135910705X27587.

[31] Janssens T, Verleden G, De Peuter S, Petersen S, Van Den Bergh O. The influence of fear of symptoms and perceived control on asthma symptom perception. J Psychosom Res 2011;71:154-159.

[32] Karos K, Meulders A, Gatzounis R, Seelen HAM, Geers RPG, Vlaeyen JWSJWSJWSJWS. Fear of pain changes movement: Motor behaviour following the acquisition of pain-related fear. Eur J Pain 2017;21:1432-1442. doi:10.1002/ejp.1044.

[33] Karos K, Meulders A, Goubert L, Vlaeyen JWS. Hide Your Pain: Social Threat Increases Pain Reports and Aggression, but Reduces Facial Pain Expression and Empathy. J Pain 
644

645

646

647

648

649

650

651

652

653

654

655

656

657

658

659

660

661

662

663

664

665

2019. doi:10.1016/j.jpain.2019.06.014.

[34] Karos K, Meulders A, Goubert L, Vlaeyen JWS. The Influence of Social Threat on Pain, Aggression, and Empathy in Women. J Pain 2018;19:291-300.

doi:10.1016/j.jpain.2017.11.003.

[35] Karos K, Meulders A, Goubert L, Vlaeyen JWS. The Influence of Social Threat on Pain, Aggression, and Empathy in Women. J Pain 2017.

[36] Karos K, Meulders A, Vlaeyen JWSJWS. Threatening Social Context Facilitates Painrelated Fear Learning. J Pain 2015;16:214-225. doi:10.1016/j.jpain.2014.11.014.

[37] Karos K, Williams AC de C, Meulders A, Vlaeyen JWS. Pain as a threat to the social self. Pain 2018;159:1. doi:10.1097/j.pain.0000000000001257.

[38] Karos K, Williams AC de C, Meulders A, Vlaeyen JWS. Pain as a threat to the social self. Pain 2018;159:1. doi:10.1097/j.pain.0000000000001257.

[39] Krahé C, Springer A, Weinman J a, Fotopoulou A. The social modulation of pain: others as predictive signals of salience - a systematic review. Front Hum Neurosci 2013;7:386. doi:10.3389/fnhum.2013.00386.

[40] Krampe RT, Smolders C, Doumas M. Leisure sports and postural control: Can a black belt protect your balance from aging? Psychol Aging 2014;29:95-102. doi:10.1037/a0035501.

[41] Kuehn BM. News from the centers for disease control and prevention. JAMA - J Am Med Assoc 2018;319:2471.

[42] Kunz M, Lautenbacher S. The faces of pain: a cluster analysis of individual differences in facial activity patterns of pain. Eur J Pain 2014;18:813-23. doi:10.1002/j.15322149.2013.00421.x. 
666 [43] Kunz M, Rainville P, Lautenbacher S. Operant conditioning of facial displays of pain.

667 Psychosom Med 2011;73:422-31. doi:10.1097/PSY.0b013e318218db3e.

668 [44] Lamana MS, Miranda J, Tobaldini G, Fischer L, Tambeli CH. Pain chronification and 669 chronic pain impair a defensive behavior, but not the ability of acute pain to facilitate it, 670 through the activation of an endogenous analgesia circuit. Behav Neurosci 2018;132:614-

671 623.

672

[45] Lee H, Lamb SE, Bagg MK, Toomey E, Cashin AG, Moseley GL. Reproducible and 673 replicable pain research. Pain 2018;159:1683. doi:10.1097/j.pain.0000000000001254.

[46] Lopes FL, Azevedo TM, Imbiriba LA, Freire RC, Valença AM, Caldirola D, Perna G, Volchan E, Nardi AE. Freezing reaction in panic disorder patients associated with anticipatory anxiety. Depress Anxiety 2009;26:917-921. doi:10.1002/da.20593.

[47] Lundqvist D, Flykt A, Öhman A. The Karolinska directed emotional faces (KDEF). CD ROM from Dep Clin Neurosci Psychol Sect Karolinska Institutet 1998:ISBN 91-6307164-9.

[48] Meulders A, Franssen M, Fonteyne R, Vlaeyen JWS. Acquisition and extinction of operant pain-related avoidance behavior using a 3 degrees-of-freedom robotic arm. Pain 2016;157:1094-1104. doi:10.1097/j.pain.0000000000000483.

[49] Meulders A, Meulders M, Vlaeyen JWS. Positive affect protects against deficient safety learning during extinction of fear of movement-related pain in healthy individuals scoring relatively high on trait anxiety. J Pain 2014;15:632-644. doi:10.1016/j.jpain.2014.02.009. related pain and associative learning: a novel pain-relevant human fear conditioning 
688

689

690

691

692

693

694

695

696

697

698

699

700

701

702

703

704

705

706

707

708

709

paradigm. Pain 2011;152:2460-9. doi:10.1016/j.pain.2011.05.015.

[51] Meulders A, Vlaeyen JWS. The acquisition and generalization of cued and contextual pain-related fear: an experimental study using a voluntary movement paradigm. Pain 2013;154:272-82. doi:10.1016/j.pain.2012.10.025.

[52] Mobbs D, Marchant JL, Hassabis D, Seymour B, Tan G, Gray M, Petrovic P, Dolan RJ, Frith $\mathrm{CD}$. From threat to fear: the neural organization of defensive fear systems in humans. J Neurosci 2009;29:12236-12243.

[53] Niermann HC, Figner B, Roelofs K. Individual differences in defensive stress-responses: the potential relevance for psychopathology. Curr Opin Behav Sci 2017;14:94-101. doi:10.1016/j.cobeha.2017.01.002.

[54] Niermann HCM, Figner B, Tyborowska A, Cillessen AHN, Roelofs K. Investigation of the Stability of Human Freezing-Like Responses to Social Threat From Mid to Late Adolescence. Front Behav Neurosci 2018;12:1-9. doi:10.3389/fnbeh.2018.00097.

[55] Niermann HCM, Ly V, Smeekens S, Figner B, Riksen-Walraven JM, Roelofs K. Infant attachment predicts bodily freezing in adolescence: evidence from a prospective longitudinal study. Front Behav Neurosci 2015;9:1-10. doi:10.3389/fnbeh.2015.00263.

[56] Noordewier MK, Scheepers DT, Hilbert LP. Freezing in response to social threat: a replication. Psychol Res 2019. doi:10.1007/s00426-019-01203-4.

[57] Nosek BA, Alter G, Banks GC, Borsboom D, Bowman SD, Breckler SJ, Buck S, Chambers CD, Chin G, Christensen G, Contestabile M, Dafoe A, Eich E, Freese J, Glennerster R, Goroff D, Green DP, Hesse B, Humphreys M, Ishiyama J, Karlan D, Kraut A, Lupia A, Mabry P, Madon T, Malhotra N, Mayo-Wilson E, McNutt M, Miguel E, 
710

711

712

713

714

715

716

717

718

719

720

721

722

723

724

725

726

727

728

729

730

731

Paluck EL, Simonsohn U, Soderberg C, Spellman BA, Turitto J, VandenBos G, Vazire S, Wagenmakers EJ, Wilson R, Yarkoni T. Promoting an open research culture. Science (80) 2015;348:1422-1425. doi:10.1126/science.aab2374.

[58] Peeters P a M, Vlaeyen JWS. Feeling more pain, yet showing less: the influence of social threat on pain. J Pain 2011;12:1255-61. doi:10.1016/j.jpain.2011.07.007.

[59] Prkachin KM. Assessing pain by facial expression: facial expression as nexus. Pain Res Manag J Can Pain Soc = J la société Can pour le Trait la douleur 2009;14:53-8. Available: http://www.ncbi.nlm.nih.gov/pubmed/19262917.

[60] Prkachin KM. The consistency of facial expressions of pain: a comparison across modalities. Pain 1992;51:297-306. doi:10.1016/0304-3959(92)90213-U.

[61] Roelofs K. Freeze for action: Neurobiological mechanisms in animal and human freezing. Philos Trans R Soc B Biol Sci 2017;372.

[62] Roelofs K, Hagenaars M a, Stins J. Facing freeze: social threat induces bodily freeze in humans. Psychol Sci 2010;21:1575-81. doi:10.1177/0956797610384746.

[63] Rosen JB. The neurobiology of conditioned and unconditioned fear: a neurobehavioral system analysis of the amygdala. Behav Cogn Neurosci Rev 2004;3:23-41.

[64] Seers T, Derry S, Seers K, Moore RA. Professionals underestimate patients' pain. Pain 2018;159:1. doi:10.1097/j.pain.0000000000001165.

[65] Shaffer F, Ginsberg JP. An Overview of Heart Rate Variability Metrics and Norms. Front Public Heal 2017;5:1-17.

[66] Simmons JP, Nelson LD, Simonsohn U. A 21 Word Solution. SSRN Electron J 2012. doi:10.2139/ssrn.2160588. 
732 [67] Spielberger CD, Gorsuch RL, Lushene RE. State-Trait Anxiety Inventory. Palo Alto, CA:

733 Consulting Psychologists Press, 1970 p.

734 [68] Spruyt A, Clarysse J, Vansteenwegen D, Baeyens F, Hermans D. Affect 4.0: A free

735 software package for implementing psychological and psychophysiological experiments.

736 Exp Psychol 2010;57:36-45. doi:10.1027/1618-3169/a000005.

737 [69] Vlaeyen JWS, Hanssen M, Goubert L, Vervoort T, Peters ML, Van Breukelen GJP,

738 Sullivan MJ, Morley S. Threat of pain influences social context effects on verbal pain

739 report and facial expression. Behav Res Ther 2009;47:774-82.

740 doi:10.1016/j.brat.2009.05.008.

741 [70] Vlaeyen JWS, Linton SJ. Fear-avoidance and its consequences in chronic musculoskeletal pain: a state of the art. Pain 2000;85:317-332. doi:10.1016/S0304-3959(99)00242-0.

[71] Vlaeyen JWS, Linton SJ. Fear-avoidance model of chronic musculoskeletal pain: 12 years on. Pain 2012:10-13. doi:10.1016/j.pain.2011.12.009.

[72] Voerman JS, Vogel I, De Waart F, Westendorp T, Timman R, Busschbach JJ V, Van De Looij-Jansen P, De Klerk C. Bullying, abuse and family conflict as risk factors for chronic pain among Dutch adolescents. Eur J Pain (United Kingdom) 2015;19:1544-1551.

[73] Volders S, Boddez Y, De Peuter S, Meulders A, Vlaeyen JWS. Avoidance behavior in chronic pain research: A cold case revisited. Behav Res Ther 2015;64:31-37. doi:10.1016/j.brat.2014.11.003. 2002;25:439-55; discussion 455-88. Available: http://www.ncbi.nlm.nih.gov/pubmed/12879700. 
754 [75] Williams ACDC, Gallagher E, Fidalgo AR, Bentley PJ. Pain expressiveness and altruistic

755 behavior : an exploration using agent-based modeling. Pain 2016;157:759-768.

756 doi:10.1097/j.pain.0000000000000443.

757 


\section{Figure 1}

\section{Overview of the Experimental Design}

Overview of the three acquisition (A1-3) and five generalization blocks (G1-5). All blocks consisted of 20 trials, 10 of which had no auditory cue or pain-US (context-alone trials). The 10 remaining trials had an auditory cue (CS+ trials), of which 5 also involved the presentation of an electrocutaneous stimulus. Acquisition blocks were run in the threatening (represented by angry facial stimulus) and safe (represented by happy facial stimulus) social context. Generalization refers to the generalization contexts (G1-5) with varying degrees of social threat $(17 \%, 33 \%, 50 \%, 67 \%$ and $83 \%)$ during the generalization blocks. Note that the facial stimuli used in this figure are merely symbolic, and that the order of the threatening and safe context in the acquisition phase, as well as the order of the generalization contexts, was intermixed and counterbalanced across participants. 


Acquisition phase
2 context (Threat, Safe) $\mathrm{x} 3$ block (A1,2,3) $\mathrm{x} 20$ trials


Figure 2

\section{Pain-US Expectancy Ratings}

Mean self-reported pain-US expectancy ratings (+ SEs) per block (A1-3) for trials with (CS+) and without (context-alone) the auditory cue, separately for the safe and threatening context. Note $-\mathrm{SE}=$ standard error term based on mixed analysis estimates; ${ }^{*}, \mathrm{p}<.05$.






\section{Figure 3}

Heart-rate Interbeat Interval

Mean interbeat interval (+ SE) per block (A1-3) in the safe and threatening context, separately for individuals low in trait anxiety (mean -1SD) and high in trait anxiety (mean +1SD). Note $-\mathrm{SE}=$ standard error term based on mixed analysis estimates; $* *, p<.01$.

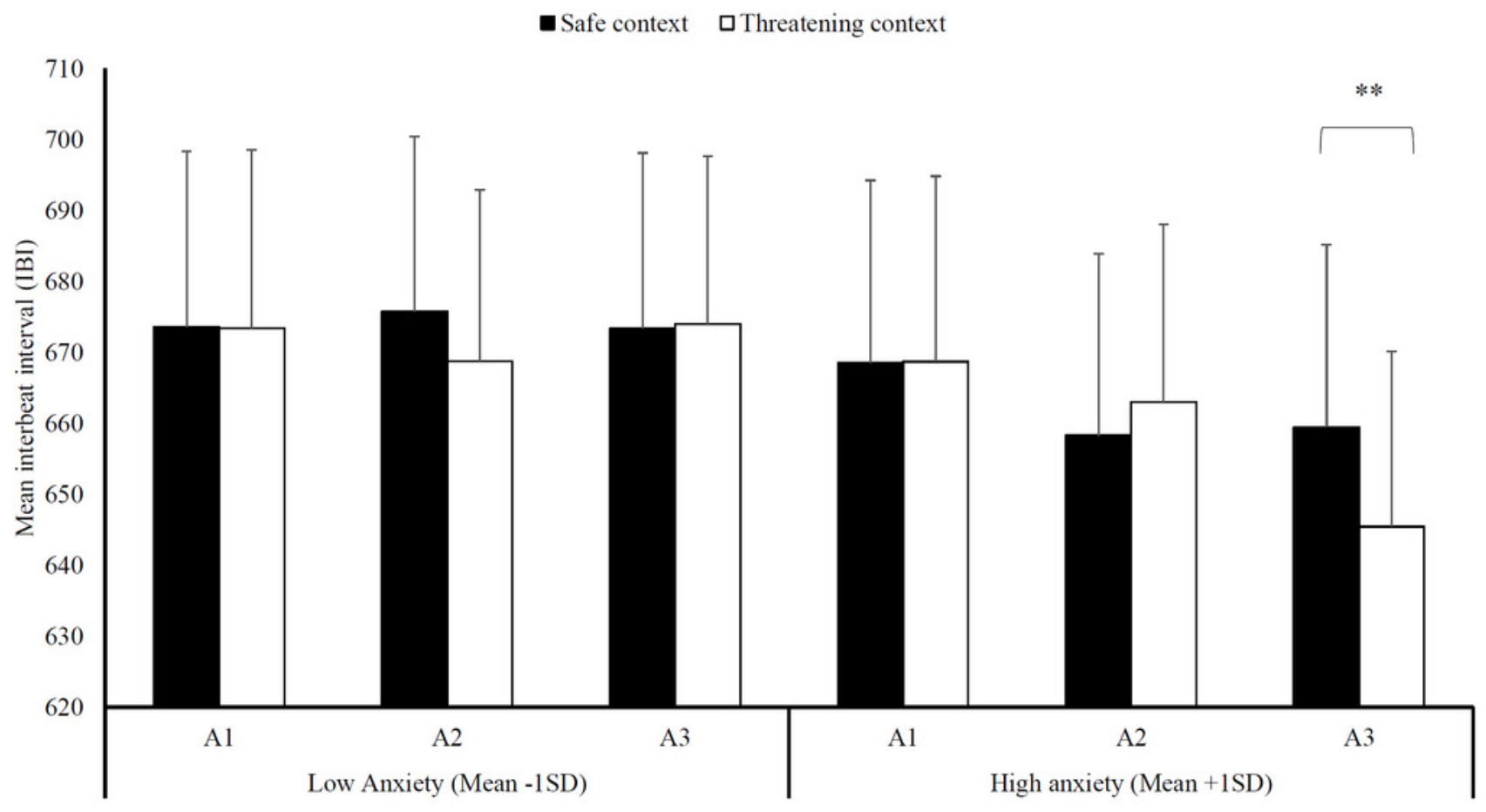




\section{Figure 4}

\section{Facial Pain Expression}

Mean facial pain expression ( $+\mathrm{SE}$ ) in the safe and threatening context, separately for individuals low in trait anxiety (mean -1SD) and high in trait anxiety (mean +1SD). Note - SE $=$ standard error term based on mixed analysis estimates. $*, p<.05$.

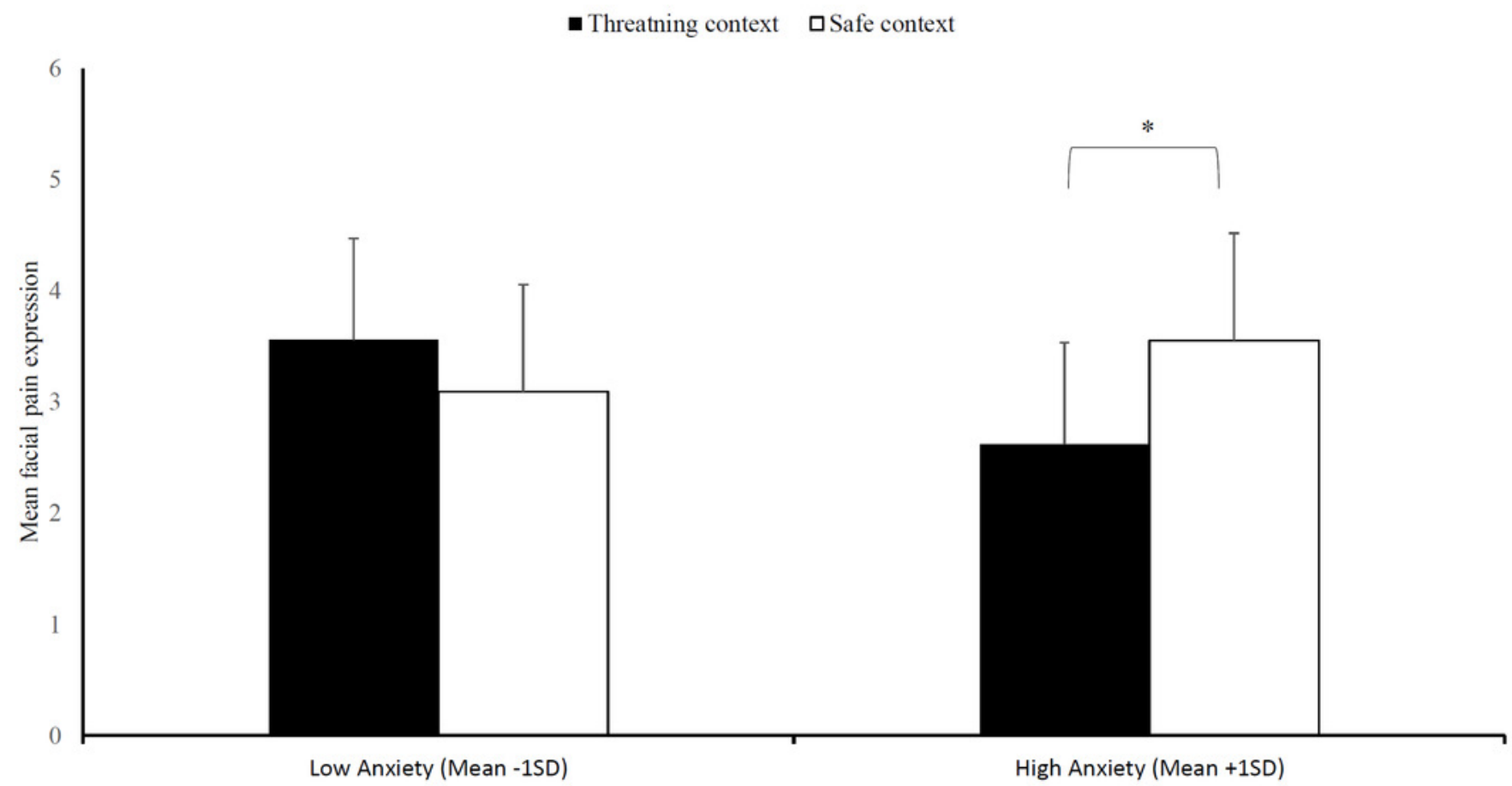

